\title{
MicroRNA-133a downregulated EGFR expression in human non-small cell lung cancer cells via AKT/ERK signaling
}

\author{
NANNAN GUO ${ }^{1 *}$, YINGNAN ZHAO $^{1 *}$, WEN ZHANG $^{1}$, SHAOJUN LI $^{1}$, SHANSHAN LI $^{2}$ and JIANQI YU ${ }^{1}$ \\ ${ }^{1}$ Department of Cardiothoracic Surgery, The First Affiliated Hospital of General Hospital of \\ The Chinese People's Liberation Army, Beijing 100048; ${ }^{2}$ Xi Shan Clinic, 309th Hospital of PLA, Beijing 100091, P.R. China
}

Received June 9, 2016; Accepted January 22, 2018

DOI: $10.3892 / 01.2018 .9399$

\begin{abstract}
MicroRNAs (miRNAs) may serve important roles in the regulation of human non-small cell lung cancer (NSCLC) cell growth and apoptosis. To the best of our knowledge, the present study was the first to explore the role of miRNA-133a/epidermal growth factor receptor (EGFR) in regulating NSCLC cell growth and apoptosis via the AKT/extracellular signal-regulated kinase (ERK) signaling pathway. It was determined that miRNA-133a expression was lower in NSCLC tissue than in the adjacent mucosae. Additionally, EGFR expression in the NSCLC tissue was higher compared with in the adjacent mucosae. Furthermore, the upregulation of miRNA-133a in NSCLC cells suppressed cell growth and induced apoptosis. Upregulating miRNA-133a also increased caspase-3 protein expression, while suppressing that of EGFR, phosphorylated (p)-AKT and p-ERK in NSCLC cells. Therefore, the results of the current study demonstrated that miRNA-133a downregulates EGFR expression in NSCLC via the AKT/ERK signaling pathway. These findings provide insights into the function of miRNA-133a in NSCLC, as well as into the molecular mechanisms underlying the miRNA-133a-mediated downregulation of the EGFR/AKT/ERK signaling pathway in NSCLC.
\end{abstract}

\section{Introduction}

Human non-small cell lung cancer (NSCLC) is a malignant tumor with a high morbidity, and is the most common cause of cancer-associated mortality worldwide (1). With improvements in living standards and public health education, the morbidity

Correspondence to: Dr Wen Zhang, Department of Cardiothoracic Surgery, The First Affiliated Hospital of General Hospital of The Chinese People's Liberation Army, 51 Fucheng Road, Haidian, Beijing 100048, P.R. China

E-mail: wengannaica3@126.com

*Contributed equally

Key words: microRNA-133a, non-small cell lung cancer, epidermal growth factor receptor, AKT, extracellular signal-regulated kinase of NSCLS has been reduced in recent years, but the morbidity for female patients with NSCLC remains the second highest among malignant tumors (2). Furthermore, the incidence of NSCLC is increasing for women. Therapeutic approaches for NSCLC are improving, but the prognosis and survival rate of NSCLC are unsatisfactory, which may be due to the lack of early diagnosis methods and treatment approaches for NSCLC, in addition to the complications associated with the disease (3). Therefore, it is necessary to explore the potential molecular mechanisms underlying the occurrence of NSCLC, in addition to novel approaches for the early diagnosis and treatment of NSCLC.

In recent years, the molecular diagnosis and targeted therapy of NSCLC have provided a new approach for the management of NSCLC (4). The use of individual genetic expression profiling and clinical staging of NSCLC in providing prognosis information has gradually become a focus for NSCLC research (5). The occurrence of NSCLC is associated with mutations, gene amplifications and epigenetic changes in tumor-associated genes (6). Epigenetic changes do not cause changes to the gene sequence, but can induce changes in the expression of tumor-associated genes, resulting in tumorigenesis and progression (6). Epigenetic research primarily focuses on the methylation of DNA, chromatin rearrangement and RNA editing changes. Additionally, miRNAs have become the focus of numerous studies in the field of cancer (7). It has been demonstrated that changes in miRNA expression are implicated in the occurrence of malignancies, at least to an extent (8).

miRNAs are small, non-coding RNAs that arise from transcriptional pri-miRNA via the activity of specific processing enzymes, including DROSHA and DICER (9). miRNA pairs with the mRNA of a target gene, resulting in mRNA degradation, or initiating gene silencing by preventing translation. Through interactions with a negative regulatory sequence in the 3'-non-coding region of specific mRNA targets, miRNA regulation is involved in a wide variety of normal and abnormal cell behaviors (10). miRNAs have potential as tumor biomarkers (11). Although the functions of a number of miRNAs have yet to be elucidated, miRNAs have been implicated in a wide range of physiological and developmental processes (10).

The present study aimed to explore the role of microRNA-133a signaling in the regulation of NSCLC 
development; to the best of our knowledge, the present study is the first to investigate this area of NSCLC research.

\section{Materials and methods}

Tissue samples. The present study was approved by the Institutional Medical Ethics Committee of The First Affiliated Hospital of the General Hospital of the Chinese People's Liberation Army (Beijing, China), and all participants $(n=8$; all male; $63 \pm 6$ years old) provided informed, written consent. Human NSCLC and adjacent normal lung tissue samples $(n=8)$ were obtained from The First Affiliated Hospital. NSCLC and adjacent normal tissues ( $>5 \mathrm{~cm}$ from tumor) were pathologically validated by a pathologist. None of the patients had received chemotherapy or radiotherapy prior to surgery.

Reverse transcription-quantitative polymerase chain reaction (RT-qPCR). Total RNA was prepared from the tissues or cells using TRIzol reagent (Bio Scientific; PerkinElmer, Inc., Waltham, MA, USA), and 1-2 ng total RNA was used for reverse transcription to cDNA using One-Step RT-PCR Kit (Takara Biotechnology Co., Ltd., Dalian, China). RT-qPCR was performed using SYBR ${ }^{\circledR}$ Green Real-Time PCR Master mix (Takara Biotechnology Co., Ltd., Dalian, China) by the Stratagene Mx3000P Real-Time PCR system (Agilent Technologies, Inc., Santa Clara, CA, USA). The specific primer pairs used are as follows: miR-133a, forward, 5'-TGC TTTGCTAGAGCTGGTAAAATG-3 and reverse, 5-AGC TACAGCTGGTTGAAGGG-3'; U6, forward, 5-CTCGCT TCGGCAGCACA-3 and reverse, 5-AACGCTTCACGAATT TGCGT-3'. The thermocycling conditions were as follows: $95^{\circ} \mathrm{C}$ for 5 min followed by 40 amplification cycles at $95^{\circ} \mathrm{C}$ for $15 \mathrm{sec}$ and $60^{\circ} \mathrm{C}$ for $30 \mathrm{sec}$. The $2^{-\Delta \Delta \mathrm{Cq}}$ method (12) was used to calculate the relative change in RNA expression as a ratio.

Immunohistochemistry. Immunohistochemistry was performed to analyze EGFR expression. Sections (thickness, $4 \mu \mathrm{m}$ ) were prepared from paraffin-embedded NSCLC and adjacent tissue samples. Sections were prepared using citrate buffer ( $\mathrm{pH}=6.0$, Sangon Biotech Co., Ltd. Shanghai, China) for $10 \mathrm{~min}$ at $95^{\circ} \mathrm{C}$ and developed with $3 \% \mathrm{H}_{2} \mathrm{O}_{2}$ in PBS for $10 \mathrm{~min}$ at room temperature. Sections were blocked with 5\% BSA (Sangon Biotech Co., Ltd.) +0.1\% TriX100 in PBS for $1 \mathrm{~h}$ at room temperature. Tissues were stained with anti-EGFR antibodies (cat no. sc-514995; 1:100 dilution; Santa Cruz Biotechnology, Inc., Dallas, TX, USA) overnight at $4^{\circ} \mathrm{C}$. Tissues were washed with PBS with $0.1 \%$ Tween-20 for $5 \mathrm{~min} 5$ times and stained with horseradish peroxidase conjugated goat anti-rabbit IgG (1:100, sc-2039; Santa Cruz Biotechnology, Inc.) for $1 \mathrm{~h}$ at room temperature and developed with 3,3'-diaminobenzidine tetrahydrochloride (Fuzhou Maixin Biotech Co., Ltd.). The slides were stained with DAB and counterstained with Mayer's hematoxylin (Beyotime Institute of Biotechnology, Inc., Haimen, China) for $1 \mathrm{~h}$.

Cells lines and reagents. The $\mathrm{H} 358$ human NSCLC cell line was purchased from the the Cell Bank of Type Culture Collection of the Chinese Academy of Sciences (Shanghai, China) and maintained in RPMI-1640 (Thermo Fisher Scientific, Inc., Waltham, MA, USA) supplemented with $10 \%$ fetal bovine serum (Gibco; Thermo Fisher Scientific, Inc.) with 100 units/ml streptomycin and 100 units $/ \mathrm{ml}$ penicillin (Thermo Fisher Scientific, Inc.) at $37^{\circ} \mathrm{C}$ in a humidified $5 \% \mathrm{CO}_{2}$ atmosphere until $75 \%$ confluent.

Transfection and lentiviral transduction. miRNA-133a mimics (forward, 5'-ACAATGTTTGCTAGAGCTG-3' and reverse, 5'-GCTGTAGCTATGCATTGA-3') and negative control (forward, 5'-CCCCCCCCCCCC-3' and 5'-CCCCCC CCCCCCCCC-3') plasmids were transfected into H358 cells using Lipofectamine 2000 transfection reagent (Thermo Fisher Scientific, Inc.) according to the manufacturer's protocol. Subsequent to transfection for 24 or $48 \mathrm{~h}$, transfected cells were used in further procedures.

MTT assay. The proliferation of transfected H358 cells was assayed at $24,48 \mathrm{~h}$ using a MTT assay kit $(50 \mu \mathrm{g} / \mathrm{ml}$; Sigma-Aldrich; Merck KGaA, Darmstadt, Germany) according to the manufacturer's protocol. Dimethyl sulfoxide $(150 \mu \mathrm{l}$; Sigma-Aldrich; Merck KGaA) was used to dissolve the formazan crystals, and the absorbance at $492 \mathrm{~nm}$ was measured using a Model 550 microplate reader (Bio-Rad Laboratories, Inc., Hercules, CA, USA).

Apoptosis assay. H358 cells transfected with miRNA-133a mimics and negative plasmids was assayed 24, $48 \mathrm{~h}$ later using an Annexin V-fluorescein isothiocyanate (FITC)/propidium iodide (PI) apoptosis assay. Annexin V-FITC/PI (BD Biosciences, San Jose, CA, USA) was added and incubated for $10 \mathrm{~min}$ at room temperature in the dark. A flow cytometer (C6; BD Biosciences, San Jose, CA, USA) was used to examine the rate of apoptosis using FlowJo software (version 7.6.1; FlowJo LLC, Ashland, OR, USA).

Caspase-3 activity assay. Transfected H358 cells were assayed at 24, $48 \mathrm{~h}$ using a Caspase-3 activity ELISA kit (cat no. C1116; Beyotime Institute of Biotechnology) for $1 \mathrm{~h}$ at $37^{\circ} \mathrm{C}$ according to the manufacturer's protocol. Caspase-3 activity was measured using a Model 550 microplate reader (Bio-Rad Laboratories, Inc.) to measure absorbance at $405 \mathrm{~nm}$.

Western blot analysis. Transfected H358 cells were assayed at $24,48 \mathrm{~h}$ using a western blot analysis. The cells were lysed with a RIPA buffer (Beyotime Institute of Biotechnology) containing a protease inhibitor cocktail (Sigma-Aldrich; Merck KGaA). The protein concentration was determinate by the Coomassie blue method. A total of $50 \mu \mathrm{g}$ protein per lane was separated with 8-15\% SDS-PAGE and blotted onto a polyvinylidene difluoride membrane (EMD Millipore, Billerica, MA, USA). Subsequent to blocking with 5\% non-fat milk in Tris-buffered saline with $0.1 \%$ Tween-20 for $1 \mathrm{~h}$ at $37^{\circ} \mathrm{C}$, blots were incubated with antibodies against caspase- 3 (cat no. sc-98785), EGFR (cat no. sc-514995), phosphorylated (p)-AKT (cat no. sc-7985-R), phosphorylation-ERK (cat no. sc-23759-R), Bax (cat no. sc-6236) and GAPDH (cat no. sc-25778) (all dilution, 1:500; Santa Cruz Biotechnology, Inc.) at $4{ }^{\circ} \mathrm{C}$ overnight, followed by incubation with anti-mouse and anti-rabbit horseradish peroxidase-conjugated secondary antibodies for $1 \mathrm{~h}$ (cat nos. sc-2005 and sc-2004; dilution, 1:5,000; Santa Cruz Biotechnology, Inc.) at $37^{\circ} \mathrm{C}$. Blots 
A

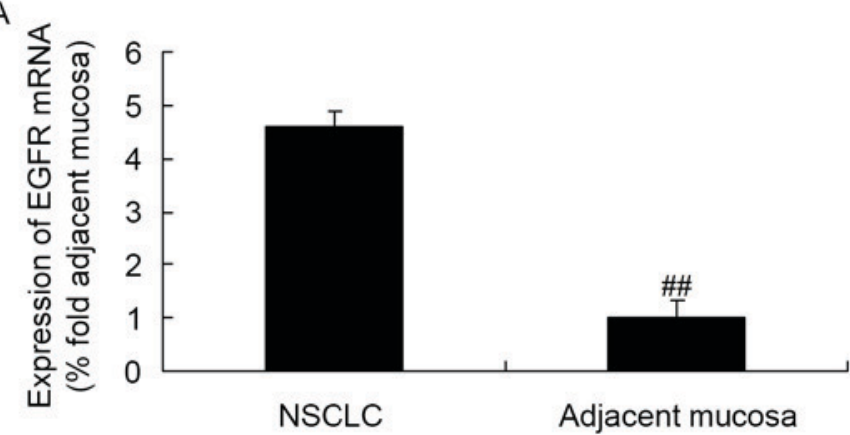

B

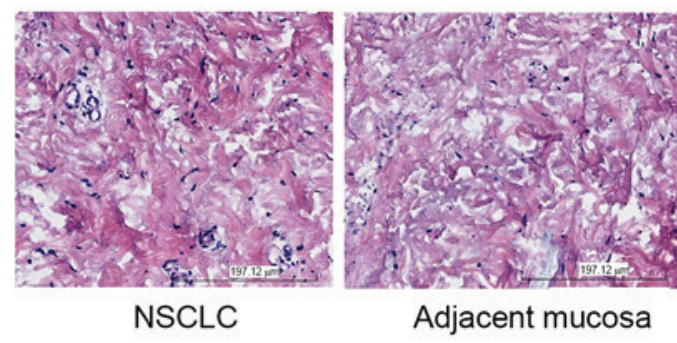

Figure 1. EGFR expression in cancer tissue of NSCLC or adjacent mucosa tissue. (A) EGFR mRNA and (B) immunohistochemical protein expression in NSCLC and adjacent mucosal tissue samples. ${ }^{\# P} \mathrm{P}<0.01$, compared with the NSCLC group. EGFR, epidermal growth factor receptor; NSCLC, non-small cell lung cancer.

were developed with an Amersham ECL Western Blotting Detection kit (GE Healthcare Life Sciences, Shanghai, China) and analyzed using Image Lab v.4.62 (Bio-Rad Laboratories, Inc.).

Statistical analysis. All values were expressed as the mean \pm standard deviation using SPSS 17.0 (SPSS, Inc., Chicago, IL, USA). Comparisons between the means of two groups were performed using Student's t-test, and with one-way analysis of variance followed by Tukey's post hoc test for multiple groups. $\mathrm{P}<0.05$ was considered to indicate a statistically significant difference.

\section{Results}

EGFR expression in NSCLC tissues and adjacent mucosae. As presented in Fig. 1A, the expression of EGFR mRNA in NSCLC tissues was higher than that in the adjacent mucosal tissues. Additionally, EGFR protein expression in the NSCLC tissues was higher than that in the adjacent mucosae (Fig. 1B).

miRNA-133a expression in NSCLC tissues and adjacent mucosae. The expression of miRNA-133a in NSCLC tissue was initially detected by RT-qPCR, which revealed that it was markedly lower than that in the adjacent mucosal tissue (Fig. 2).

NSCLC cell growth rate following miRNA-133a upregulation. To test the hypothesis that miRNA-133a may be involved in the alternative regulation of NSCLC growth, H358 cells were transfected with miRNA-133a mimics, and the expression of miRNA-133a was assessed via RT-qPCR. The miRNA-133a mimics significantly increased miRNA-133a expression in H358 cells, compared with in the control group (Fig. 3A). Furthermore, upregulated miRNA-133a significantly suppressed H358 cell growth, compared with that of the control group (Fig. 3B).

NSCLC apoptosis following miRNA-133a upregulation. NSCLC apoptosis was evaluated using an Annexin V-FITC/PI apoptosis assay, in order to assess the effect of upregulated miRNA-133a. The results showed that increased miRNA-133a significantly induced H358 cell apoptosis, as compared with the control group (Fig. 4).

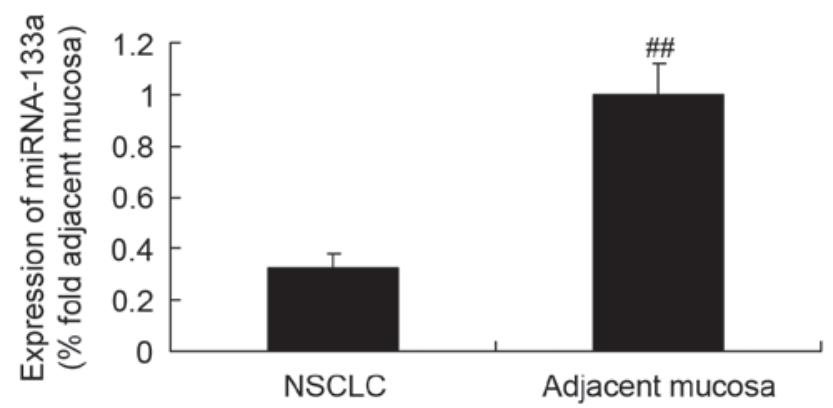

Figure 2. miRNA-133a expression in NSCLC or adjacent mucosal tissues. ${ }^{\# \prime} \mathrm{P}<0.01$, compared with the NSCLC group. miRNA, microRNA; NSCLC, non-small cell lung cancer.

Caspase-3 activity, protein expression and EGFR protein expression in NSCLC following miRNA-133a upregulation. To determine the levels of caspase-3 activity, protein expression and EGFR protein expression in NSCLC following the upregulation of miRNA-133a, a caspase-3 ELISA and western blot analysis were conducted. As depicted in Fig. 5, there were significant increases in caspase-3 activity, protein expression and EGFR protein expression in H358 cells following miRNA-133a elevation, as compared with those in the control group.

p-AKT, p-ERK and Bax protein expression in NSCLC following miRNA-133a upregulation. To investigate the association between p-AKT, p-ERK and Bax and miRNA-133a upregulation in $\mathrm{H} 358$ cells, $\mathrm{p}$-AKT, p-ERK and Bax protein expressions was analyzed via western blotting. As presented in Fig. 6, miRNA-133a upregulation significantly suppressed p-AKT, p-ERK and Bax protein expression in H358 cells, as compared with in the control group.

\section{Discussion}

NSCLC is the most common cause of cancer-associated mortality. Although methods for the diagnosis and treatment of NSCLC have improved at a constant rate, the prognosis for patients with NSCLC remains poor (13). Clinical staging of NSCLC via pathological analysis is the recommended method for evaluating NSCLC prognosis and selecting the optimal treatment approach (14). With an increasing number 

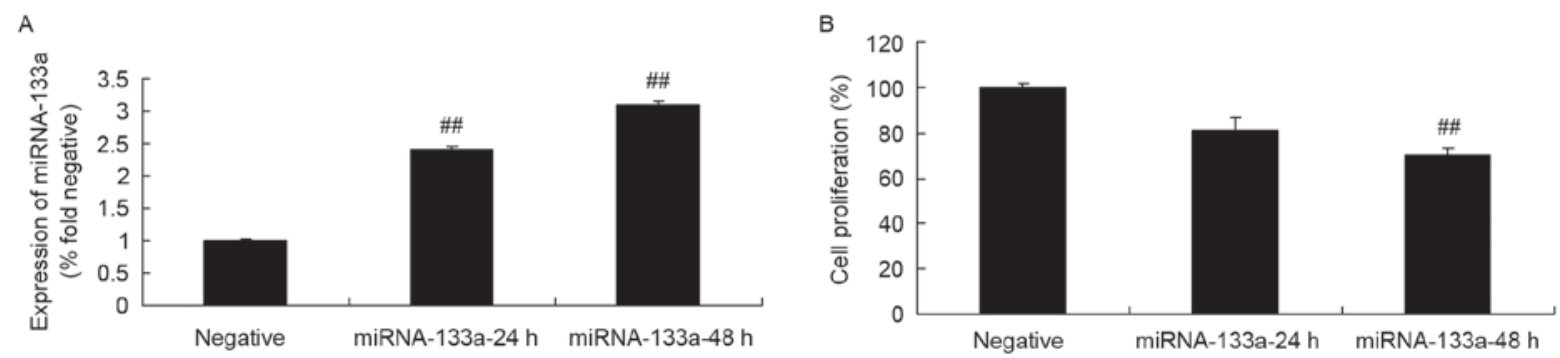

Figure 3. NSCLC cell growth rate following the upregulation of miRNA-133a. (A) miRNA-133a expression and (B) NSCLC cell growth rate following the upregulation of miRNA-133a. ${ }^{\# \#} \mathrm{P}<0.01$, compared with the NSCLC group. NSCLC, non-small cell lung cancer; miRNA, microRNA.

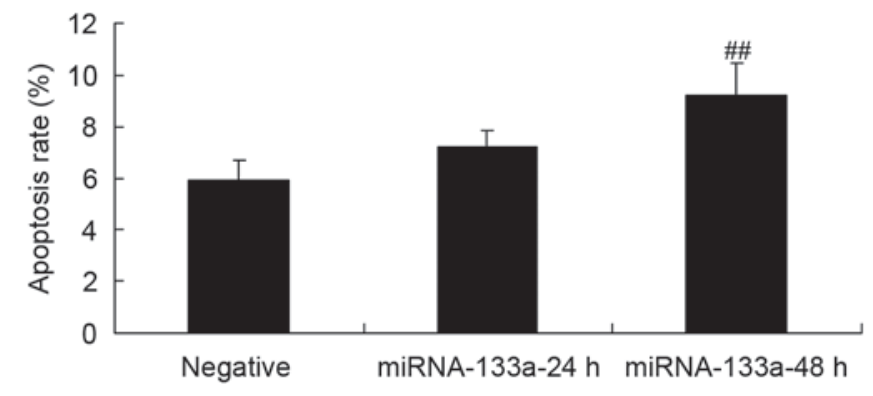

Figure 4. NSCLC cell apoptosis rate following the upregulation of miRNA-133a. ${ }^{\# \#} \mathrm{P}<0.01$, compared with the NSCLC group. NSCLC, non-small cell lung cancer; miRNA, microRNA.

of in-depth studies of the effects of miRNA in tumors and other diseases, miRNA expression changes in NSCLC are gradually becoming a hotspot in this area of research. When classifying the subtype of a tumor, the miRNA expression profile of the tumor tissue can be more accurate than using mRNA (15). Studies have indicated that miRNAs are abnormally expressed in NSCLC, but there is no consensus on the miRNAs involved $(8,15)$. A wide range of different alterations to miRNA expression have been identified (15). Potentially, miRNA analysis can be integrated into staging methods for the diagnosis and prognosis of NSCLC, and potentially allow individualized NSCLC treatment (16). In the present study, the expression of miRNA-133a in NSCLC tissue was markedly lower than that of the adjacent mucosa tissue. Furthermore, upregulating miRNA-133a significantly suppressed cell growth, induced apoptosis, and increase caspase- 3 activity and protein expression in $\mathrm{H} 358$ cells. These data support that miRNA-133 is a tumor suppressor in lung cancer.

A total of $90 \%$ the identified EGFR gene mutations in NSCLC are located in exons 19-21 (17). EGFR can be expressed in epithelial, mesenchymal and neurogenic tissues and it serves a important role in regulating hyperplasia, growth and the differentiation of normal cells (17). EGFR also induces the growth of tumor cells, vascularization, tumor metastasis and apoptosis resistance (18). Once a ligand interacts with the EGFR-N extracellular domain, an EGFR homo- or heterodimer can be formed, resulting in the phosphorylation of intracellular tyrosine residues and the activation of downstream signal pathways, including the RAS/RAF/ERK/MAPK pathway, the P13K/AKT pathway and the STAT3/5 signal transduction pathways. The result is the expression of genes that promote tumorigenic cell behavior, including proliferation, invasion, metastasis, angiogenesis and dysplasia (18). The dimer formed upon the EGFR-N extracellular binding domain and ligand binding is the requirement for the phosphorylation of EGFR tyrosine residues and the activation of downstream signaling (19). In the present study, it was identified that upregulating miRNA-133a significantly suppressed the EGFR expression of H358 cells. Cui et al (20) identified that microRNA-133a regulated the proliferation of breast cancer through the EGFR/Akt signaling pathway. Song et al (21) demonstrated that miR-133a suppressed cervical cancer growth through the Akt and ERK signaling pathways. The data presented in the present study suggest that EGFR serves an important role in miRNA-133-induced lung cancer suppression.

Tumor cells commonly exhibit altered signal transduction and imbalanced cell growth, differentiation and apoptosis (22). PI3K/AKT signal transduction is an important intracellular signal transduction pathway that can induce the occurrence and development of numerous types of tumor by affecting cell cycle control, cell survival, metastasis, angiogenesis and chemotherapy resistance (23). Activation often occurs in the early stages of oncogenesis and tumor progression. The degree of signal pathway activation is an important indicator of the prognosis of patients with tumors (24). In the present study, the upregulation of miRNA-133a significantly suppressed the p-AKT protein expression in H358 cells. Cui et al (20) demonstrated that microRNA-133a regulated the proliferation of breast cancer cells through the EGFR/Akt signaling pathway. This is in accord with our novel finding that the regulation of Akt serves a role in the effect of miRNA-133a on lung cancer.

The ERK pathway is an important signal pathway in the occurrence and development of NSCLC, and is a vital target of anticancer treatment (25). Transcription factor AP-2 $\beta$ overexpression has been associated with the incidence of lung adenocarcinoma, which is associated with the regulation of the ERK pathway. Mitogen-inducible gene 6 expression downregulation inhibits NSCLC cell apoptosis, activates the ERK pathway and upregulates the anti-apoptotic protein Bcl-2 (26). ERK activation mediated by Src can promote the gefitinib resistance of NSCLC cells (26). Inhibition of the ERK pathway can prevent NSCLC cells from undergoing epithelial-mesenchymal transition and promote their sensitivity to EGFR inhibitors (27). AKT and ERK pathways also interact to promote NSCLC cell proliferation and survival. Activating the AKT and ERK pathways simultaneously induces drug resistance in NSCLC cells (28). Mucin 1 mucoprotein may induce the ERK2 pathway to regulate cell growth and differentiation (28). In the present study, the upregulation 


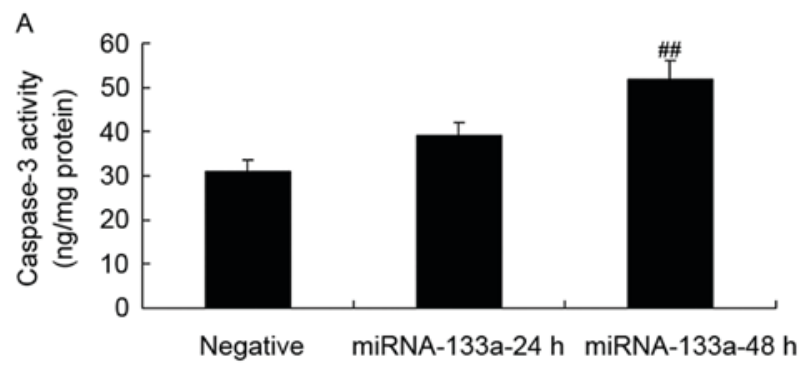

B
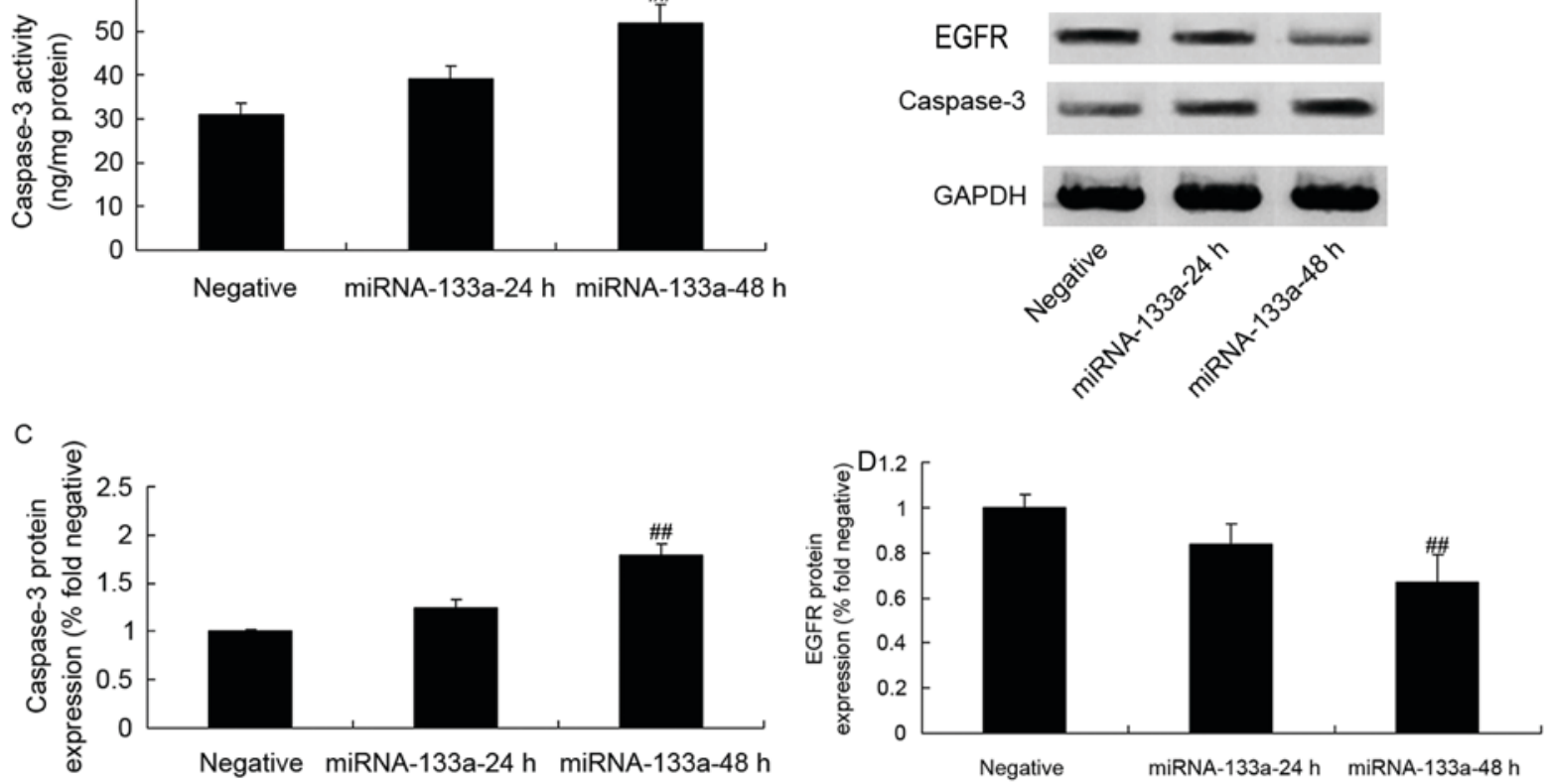

Figure 5. Caspase-3 activity, protein expression and EGFR protein expression in NSCLC cells following the upregulation of miRNA-133a. (A) Caspase-3 activity, Caspase-3 and EGFR protein expression determined using (B) western blotting that was (C and D) quantified for (C) Caspase-3 and (D) EGFR protein expression in NSCLC cells following the upregulation of miRNA-133a. ${ }^{\# \#} \mathrm{P}<0.01$, compared with the NSCLC group. NSCLC, non-small cell lung cancer; miRNA, microRNA; EGFR, epidermal growth factor receptor.
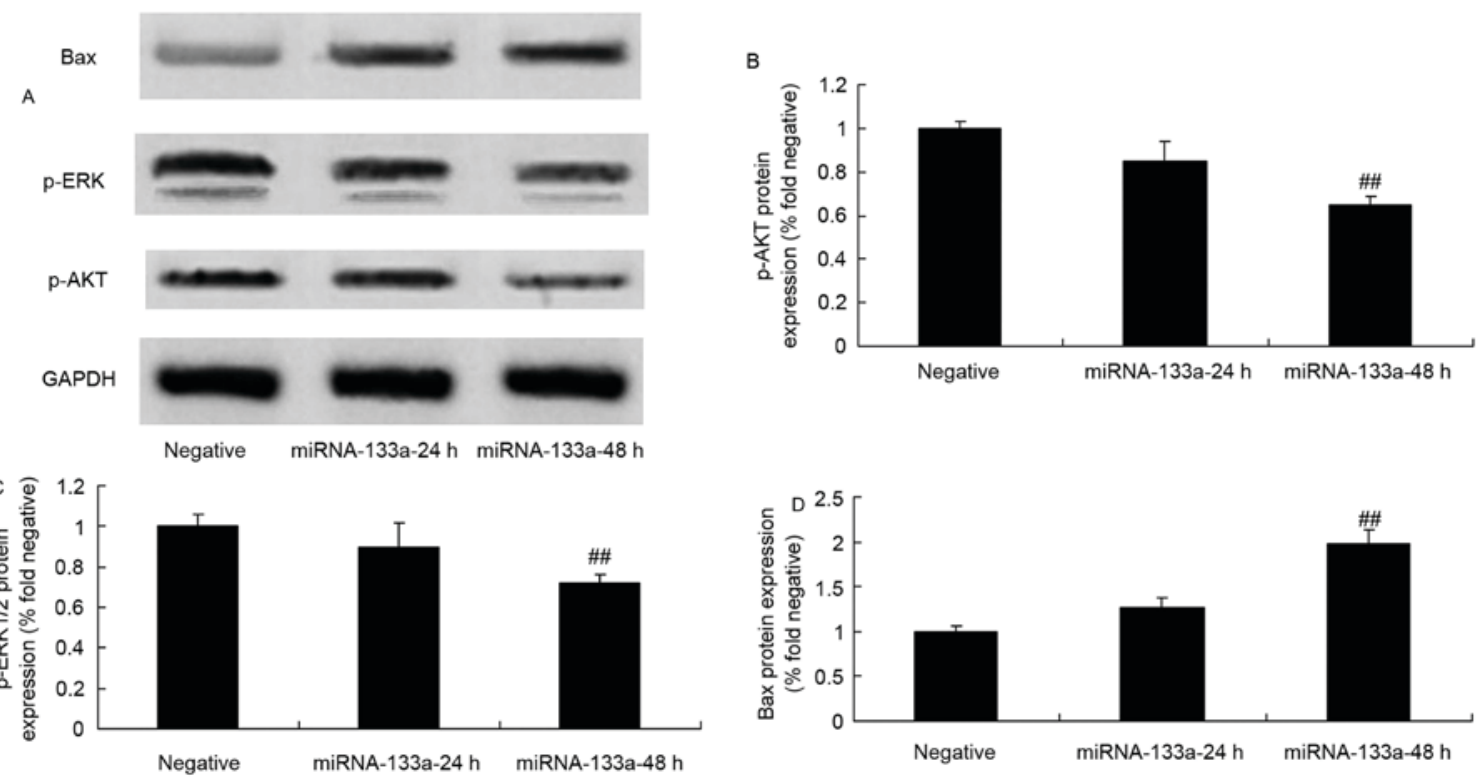

Figure 6. p-AKT, p-ERK and Bax protein expression in NSCLC cells following the upregulation of miRNA-133a. p-AKT, p-ERK and Bax expression in NSCLC tissues was evaluated via (A) western blot that was quantified for (B) p-AKT, (C) p-ERK and (D) Bax expression, following the upregulation of miRNA-133a. ${ }^{\# \#} \mathrm{P}<0.01$, compared with the NSCLC group. NSCLC, non-small cell lung cancer; miRNA, microRNA; p-, phosphorylated; ERK, phosphorylated extracellular signal-regulated kinase; Bax, Bcl-2-associated X; AKT, protein kinase B.

of miRNA-133a significantly inhibited p-ERK protein expression and induced the expression of Bax protein in H358 cells. Song et al (21) demonstrated that miR-133a suppresses cervical cancer growth through an effect on the AKT and ERK signaling pathways.

In conclusion, the present study has demonstrated that the upregulation of miRNA-133a significantly suppressed cell growth, induced apoptosis, and increased caspase-3 activity and protein expression via the EGFR/AKT/ERK signaling pathway in H358 NSCLC cells. Thus, targeting miRNA-133a may exhibit potential as a novel therapeutic strategy against NSCLC through the EGFR/AKT/ERK signaling pathway.

\section{Acknowledgements}

Not applicable. 


\section{Funding}

Not applicable.

\section{Availability of data and materials}

The analyzed data sets generated during the study are available from the corresponding author, on reasonable request.

\section{Authors' contributions}

WZ designed the experiment. NNG, YNZ, SJL, SSL and JQY performed the experiment. WZ and JQY analyzed the data. WZ wrote the manuscript.

\section{Ethics approval and consent to participate}

The present study was approved by the Institutional Medical Ethics Committee of The First Affiliated Hospital of the General Hospital of the Chinese People's Liberation Army, and all participants provided informed, written consent.

\section{Patient consent for publication}

Not applicable.

\section{Competing interests}

The authors declare that they have no competing interests.

\section{References}

1. Videtic GM, Hu C, Singh AK, Chang JY, Parker W, Olivier KR, Schild SE, Komaki R, Urbanic JJ, Timmerman RD and Choy H: A randomized phase 2 study comparing 2 stereotactic body radiation therapy schedules for medically inoperable patients with stage i peripheral non-small cell lung cancer: NRG oncology RTOG 0915 (NCCTG N0927). Int J Radiat Oncol Biol Phys 93: 757-764, 2015

2. Nie X, Cheng G, Ai B and Zhang S: The tailored chemotherapy based on RRM1 immunohistochemical expression in patients with advanced non-small cell lung cancer. Cancer Biomark 13 433-440, 2013.

3. Karp DD, Lee SJ, Keller SM, Wright GS, Aisner S, Belinsky SA, Johnson DH, Johnston MR, Goodman G, Clamon G, et al: Randomized, double-blind, placebo-controlled, phase III chemoprevention trial of selenium supplementation in patients with resected stage I non-small-cell lung cancer: ECOG 5597. J Clin Oncol 31: 4179-4187, 2013.

4. Wang R, Chen XF and Shu YQ: Prediction of non-small cell lung cancer metastasis-associated microRNAs using bioinformatics. Am J Cancer Res 5: 32-51, 2014.

5. Sin TK, Wang F, Meng F, Wong SC, Cho WC, Siu PM, Chan LW and Yung BY: Implications of MicroRNAs in the treatment of gefitinib-resistant non-small cell lung cancer. Int J Mol Sci 17: $237,2016$.

6. Chen F, Hou SK, Fan HJ and Liu YF: MiR-15a-16 represses Cripto and inhibits NSCLC cell progression. Mol Cell Biochem 391: $11-19,2014$

7. Wu N, Zhang C, Bai C, Han YP and Li Q: MiR-4782-3p inhibited non-small cell lung cancer growth via USP14. Cell Physiol Biochem 33: 457-467, 2014

8. Lin R, Chen L, Chen G, Hu C, Jiang S, Sevilla J, Wan Y, Sampson JH, Zhu B and Li QJ: Targeting miR-23a in CD8+ cytotoxic T lymphocytes prevents tumor-dependent immunosuppression. J Clin Invest 124: 5352-5367, 2014.

9. Li N, Zhang F, Li S and Zhou S: Epigenetic silencing of MicroRNA-503 regulates FANCA expression in non-small cell lung cancer cell. Biochem Biophys Res Commun 444: 611-616, 2014.
10. Wang X, Ling C, Bai Y and Zhao J: MicroRNA-206 is associated with invasion and metastasis of lung cancer. Anat Rec (Hoboken) 294: 88-92, 2011.

11. Aghanoori MR, Mirzaei B and Tavallaei M: MiRNA molecular profiles in human medical conditions: Connecting lung cancer and lung development phenomena. Asian Pac J Cancer Prev 15: 9557-9565, 2014.

12. Livak KJ and Schmittgen TD: Analysis of relative gene expression data using real-time quantitative PCR and the 2(-Delta Delta C(T)) method. Methods 25: 402-408, 2001.

13. Dy GK, Hillman SL, Rowland KM Jr, Molina JR, Steen PD, Wender DB, Nair S, Mandrekar S, Schild SE and Adjei AA; North Central Cancer Treatment Group Study N0326: A front-line window of opportunity phase 2 study of sorafenib in patients with advanced nonsmall cell lung cancer: North central cancer treatment group study N0326. Cancer 116: 5686-5693, 2010.

14. Mita AC, Papadopoulos K, de Jonge MJ, Schwartz G, Verweij J, Mita MM, Ricart A, Chu QS, Tolcher AW, Wood L, et al: Erlotinib 'dosing-to-rash': A phase II intrapatient dose escalation and pharmacologic study of erlotinib in previously treated advanced non-small cell lung cancer. Br J Cancer 105: 938-944, 2011.

15. Roa WH, Kim JO, Razzak R, Du H, Guo L, Singh R, Gazala S, Ghosh S, Wong E, Joy AA, et al: Sputum microRNA profiling: A novel approach for the early detection of non-small cell lung cancer. Clin Invest Med 35: E271, 2012.

16. Zhang W, Winder T, Ning Y, Pohl A, Yang D, Kahn M, Lurje G, Labonte MJ, Wilson PM, Gordon MA, et al: A let-7 microRNA-binding site polymorphism in 3'-untranslated region of KRAS gene predicts response in wild-type KRAS patients with metastatic colorectal cancer treated with cetuximab monotherapy. Ann Oncol 22: 104-109, 2011.

17. Karachaliou N, Rosell R, Morales-Espinosa D and Viteri S: Systemic treatment in EGFR-ALK NSCLC patients: Second line therapy and beyond. Expert Rev Anticancer Ther 14: 807-815, 2014.

18. Janmaat ML, Rodriguez JA, Gallegos-Ruiz M, Kruyt FA and Giaccone G: Enhanced cytotoxicity induced by gefitinib and specific inhibitors of the Ras or phosphatidyl inositol-3 kinase pathways in non-small cell lung cancer cells. Int J Cancer 118: 209-214, 2006.

19. Raben D, Helfrich B and Bunn PA Jr: Targeted therapies for non-small-cell lung cancer: Biology, rationale, and preclinical results from a radiation oncology perspective. Int J Radiat Oncol Biol Phys 59 (2 Suppl): S27-S38, 2004.

20. Cui W, Zhang S, Shan C, Zhou L and Zhou Z: microRNA-133a regulates the cell cycle and proliferation of breast cancer cells by targeting epidermal growth factor receptor through the EGFR/Akt signaling pathway. FEBS J 280: 3962-3974, 2013.

21. Song X, Shi B, Huang K and Zhang W: miR-133a inhibits cervical cancer growth by targeting EGFR. Oncol Rep 34: 1573-1580, 2015.

22. Zhen Y, Li D, Li W, Yao W, Wu A, Huang J, Gu H, Huang Y, Wang Y, Wu J, et al: Reduced PDCD4 expression promotes cell growth through PI3K/Akt signaling in non-small cell lung cancer. Oncol Res 23: 61-68, 2016.

23. Cheng H, Shcherba M, Pendurti G, Liang Y, Piperdi B and Perez-Soler R: Targeting the PI3K/AKT/mTOR pathway: Potential for lung cancer treatment. Lung Cancer Manag 3: 67-75, 2014.

24. Dong M, Yang G, Liu H, Liu X, Lin S, Sun D and Wang Y: Aged black garlic extract inhibits HT29 colon cancer cell growth via the PI3K/Akt signaling pathway. Biomed Rep 2: 250-254, 2014.

25. Wang H, Wu C, Wan S, Zhang H, Zhou S and Liu G: Shikonin attenuates lung cancer cell adhesion to extracellular matrix and metastasis by inhibiting integrin $\beta 1$ expression and the ERK1/2 signaling pathway. Toxicology 308: 104-112, 2013.

26. Zinn RL, Gardner EE, Marchionni L, Murphy SC, Dobromilskaya I, Hann CL and Rudin CM: ERK phosphorylation is predictive of resistance to IGF-1R inhibition in small cell lung cancer. Mol Cancer Ther 12: 1131-1139, 2013.

27. Zhao S, Wu J, Zheng F, Tang Q, Yang L, Li L, Wu W and Hann SS: $\beta$-elemene inhibited expression of DNA methyltransferase 1 through activation of ERK1/2 and AMPK $\alpha$ signalling pathways in human lung cancer cells: the role of Sp1. J Cell Mol Med 19: 630-641, 2015.

28. Gao J, Zhao Y, Lv Y, Chen Y, Wei B, Tian J, Yang Z, Kong F, Pang J, Liu J and Shi H: Mirk/Dyrk1B mediates G0/G1 to S phase cell cycle progression and cell survival involving MAPK/ERK signaling in human cancer cells. Cancer Cell Int 13: 2, 2013. 